\title{
Systemic lupus erythematosus in pregnancy - intricate, but wieldy
}

\author{
Ritin Mohindra ${ }^{1}$, Sheeba Marwah ${ }^{2}$ \\ ${ }^{1}$ Department of Internal Medicine, VMMC and Safdarjung Hospital, New Delhi, India \\ ${ }^{2}$ Department of Obstetrics \& Gynecology, VMMC and Safdarjung Hospital, New Delhi, India
}

Received: 01 February 2015

Accepted: 01 March 2015

\author{
*Correspondence: \\ Dr. Ritin Mohindra, \\ E-mail:ritin.mohindra@gmail.com
}

Copyright: $\odot$ the author(s), publisher and licensee Medip Academy. This is an open-access article distributed under the terms of the Creative Commons Attribution Non-Commercial License, which permits unrestricted non-commercial use, distribution, and reproduction in any medium, provided the original work is properly cited.

\begin{abstract}
Systemic Lupus Erythematosus (SLE) predominantly afflicts young women in reproductive age. In this context, it is only rational that pregnancy and its outcome becomes an imperative concern for the lupus patients and their health care providers. Queries regarding the risk of disease flares during pregnancy, chance of fetal loss, and the safety of various drugs are often raised. However the present day availability of effective treatment regimens has meant that many patients achieve protracted remissions and better disease control. Indeed, a high proportion of lupus patients, with professional care, can thus look forward to a successful pregnancy. ${ }^{1}$ This article reviews the contemporary concepts regarding SLE and pregnancy, especially regarding the fertility rate, optimal timing of conception, risk of disease flares during lupus pregnancy, pregnancy course, fetal outcome, safety of various drugs used for disease control during pregnancy and lactation, and contraceptive advice.
\end{abstract}

Keywords: Systemic lupus erythematosus, Lupus, Pregnancy, Fetal outcome, Maternal outcome

\section{INTRODUCTION}

SLE is a chronic multisystem autoimmune connective tissue disorder that primarily affects women of childbearing age. With the improvement in the understanding of the pathogenesis of SLE and the judicious use of immunosuppressive drugs leading to improvement in survival and prognosis of SLE recently, better disease control can now be achieved, and SLE patients should not be deprived of the opportunity for bearing children. However, uncertainties about the risk of childbearing to both the mother and the fetus have emerged as the chief apprehension of such couples, during clinic consultations. Advising them on various aspects of pregnancy and contraception is always an arduous task. In this review article, common issues related to pregnancy in women with SLE are conferred. Apposite distinct individual based advice should be given to couples during pre-pregnancy counselling, in close alliance with medicine specialists, rheumatologists and perinatologists, in order to optimise the maternal and fetal outcome.

\section{EFFECT OF PREGNANCY ON SLE}

The effect of pregnancy on maternal disease is controversial. While some surveys have depicted exacerbation of SLE during pregnancy, ${ }^{1,2}$ others have not. $^{3,4}$ The disparity in results from these studies is multifactorial and probably can be attributed to the differences in the definition of lupus flares, assessment of disease activity, selection of the control groups, and whether they matched well the disease characteristics of the pregnant patients, the proportion of patients with positive antiphospholipid antibodies (aPL) and abortion during early pregnancy who were excluded from analysis, and therapeutic strategies such as prophylactic steroids in late pregnancy. 


\section{PREGNANCY AND LUPUS FLARES}

Notwithstanding the varying reports available in literature, most rheumatologists agree that lupus flares are frequent in pregnancy, with a flare rate of 0.06-0.136 per patient-month. Flares are expected during pregnancy because of increased levels of estrogen, prolactin, and certain cytokines and prediction of when this will happen during the pregnancy course is difficult. Flares can occur during any trimester or post-partum, necessitating close follow up. ${ }^{2-6}$ Women undergoing in vitro fertilization may also have an increased risk of a disease flare during ovulation induction. Risk factors for post-gestational flares include a high pregestational Systemic Lupus Erythematosus Disease Activity Index (SLEPDAI), low serum albumin, elevated anti-double-stranded DNA antibody titres, proteinuria and use of steroids during pregnancy. ${ }^{7}$ On the other hand, a long time of remission before pregnancy decreases the risk of aggravation. Every SLE patient should therefore be followed up during pregnancy under the assumption that there is a risk of flare.

The clinician should be alive to the fact that assessment of lupus activity during pregnancy can be difficult. Physiological changes like alopecia, transient facial blush palmar erythema, chloasma, increase in proteinuria due to increased glomerular filtration rate, and postpartum alopecia are likely to be misconstrued as flares of the disease by the unwary. ${ }^{8}$ Also before labelling an aggravation, SLE unrelated complications of pregnancy presenting in a similar fashion, need to be excluded. Most pertinent of these are proteinuria from preeclampsia, thrombocytopenia of the HELLP syndrome. Therefore lupus disease activity indices have been modified in pregnancy in an effort to pick up lupus flares accurately. Such indices include Systemic Lupus Erythematosus Disease Activity Index (SLEPDAI), the Lupus Activity Index in Pregnancy (LAI-P), and the modified Systemic Lupus Activity Measure (m-SLAM).

The flares are generally mild, with pregnant women experiencing arthritis, fatigue and cutaneous manifestations. Exacerbation of SLE with major organ involvement such as the central nervous system and the kidneys, on the other hand, may occur in upto 5\% and $46 \%$, patients respectively. ${ }^{2-6,9}$ Thus patients with preexisting major organ involvement need close monitoring for disease activity during pregnancy and the puerperium.

Flares are managed by escalation of treatment like NonSteroidal Anti-Inflammatory Drugs (NSAIDs) and corticosteroids. Major organ flares may need dose escalation of azathioprine. Cyclophosphamide is contraindicated in pregnancy. Prophylactic corticosteroids to prevent lupus flares during pregnancy are not recommended. ${ }^{4}$ Overall, studies do not suggest lupus flares during pregnancy are exceedingly more serious than those occurring otherwise.

\section{EFFECT OF SLE ON PREGNANCY}

\section{Pre-conceptional counselling}

Better management and multidisciplinary care have meant that successful pregnancies are becoming the rule rather than exception in SLE. Thus adequate preconception counselling must be provided by the rheumatologist during the initial visits for all women of childbearing age, and a more formal preconceptual consultation with a maternal-fetal specialist is recommended when the patient expresses a desire to become pregnant. The goal of preconception counselling should be to develop a bond with the patient in pursuit of optimizing her health before pregnancy. The timing of conception is an important determinant in pregnancy outcome for lupus. All lupus pregnancies should be considered high risk and closely monitored. Patient education is also critical for a successful pregnancy outcome. Lab evaluation for assessment of anemia, thrombocytopenia, renal function and APL antibodies must be done. Before attempting to conceive, teratogenic immunosupressants should be swapped with those most apt for pregnancy, provided lupus disease activity is quiescent. However, if the patient requires immunosuppressive therapy for maintaining disease control, she should be kept on a regimen with the lowest fetal risk.

\section{Optimal timing for conception, and contraception}

Planned pregnancies are preferred in lupus patients. In an intended conception that occurs when the disease has been in remission for 6 months prior, fetal and maternal outcomes have been found to be more favourable. If pregnancies occur when the lupus disease is active, or a major organ involvement, or lupus nephritis, they are more likely to result in poor fetal and maternal outcomes. Thus patients need to be counselled about various contraceptive options.

All reversible methods are acceptable except oral contraceptive pills, the barrier contraception being the safest pick. ${ }^{10}$ Intra-uterine devices carry an increased risk of infection, especially in women on immunosupressants, and are better avoided. The currently available oral pills with very low dose of estrogens are probably safe in SLE patients (with close monitoring for disease activity and signs of thromboembolism), barring antiphospholipid syndrome (APS). However, progesterone only pills or depot progestogens are also innocuous in lupus, although side effects like menstrual irregularities, spotting, amenorrhea, edema, weight gain may adversely affect patient acceptance.

Permanent sterilization must be considered in progressive \& unrelenting cases. Besides, women who have completed their families can safely undergo tubal ligation. 


\section{Fertility}

Fecundity in patients with SLE usually remains inviolate by the disease. Pregnancy rates of 2-2.4 pregnancies per patient have been described, ${ }^{11}$ both during disease remission and activity. Lower fertility is observed in a small subset of patients with end-stage renal disease (amenorrhea), cyclophosphamide treatment (ovarian failure), and due to active disease or high dose corticosteroids (anovulatory cycles). Intravenous pulse cyclophosphamide given intermittently is better than daily oral cyclophosphamide for preservation of gonadal function. The chances of permanent amenorrhea are higher with the latter. Either the use of OCPs to put ovaries to repose, or prescribing gonadotropin releasing hormones, can preserve fertility in lupus patients undergoing cyclophosphamide treatment. ${ }^{10}$ Infertile women with lupus can be considered for In Vitro Fertilization (IVF). Estrogens given as part of IVF regimens might intensify SLE, but most flares are manageable, and hence are not denied in these selected women.

\section{Obstetric issues during pregnancy}

The risk of maternal morbidity is increased in the setting of active lupus nephritis (LN), including an increased risk of hypertensive disorders of pregnancy. Lupus patients have an increased risk of preeclampsia $(5-38 \%)$ as compared to women without SLE. ${ }^{11}$ Risk factor for preeclampsia include pre-existing hypertension, nephritis and presence of antiphospholipid antibodies (aPL). Challenge is to clinically differentiate between preeclampsia and nephritic flare since both conditions lead to hypertension, proteinuria, edema and deterioration in renal function. However a drop in complement levels coupled with risinganti- ds-DNA titers, with possible absence of hypertension and higher active urinary sediments (white cell, red cell, or granular casts) help delineating lupus cases. ${ }^{12}$ Also disease activity in other organs such as true arthritis, cutaneous vasculitis, oral ulcers, and lymphadenopathy in a patient with worsening proteinuria points to a lupus flare. ${ }^{12,13}$ While such patients respond well to prednisone treatment, latter will typically worsen preeclampsia. But, preeclampsia and renal lupus may coexist in the same patient. Although progressive renal impairment is the rule, it's often mild, and ESRD requiring hemodialysis is rare, even in patients with active LN. The treatment rubrics for preeclampsia and eclampsia are the same as in a non-lupus patient.

\section{The effect of lupus on fetal outcomes}

Research on lupus has construed a higher rate of abortions (6-35\%), still-births (0-22\%), and prematurity and Intra-Uterine Growth Retardation (IUGR) in pregnant women. The proven predictive factors for fetal wastage comprise of active LN, hypercoagulability (placental infarctions and consequent fetal hypoxia), placental pathology (restricted blood flow), previous history of fetal death and presence of aPL. ${ }^{14}$ Unfavourable live birth outcomes correlate with lower pre-gestational serum albumin levels, elevated gestational anti-double-stranded DNA antibody titters and diabetes. Fetal growth restriction can occur even before the lupus is apparent in the mother which suggests a pre-disease state. Maternal hypertension and high dose periconceptional use of steroids, severe flare during pregnancy, nephrotic range proteinuria and anticardiolipin antibody (aCL) are prognosticators for prematurity and IUGR. Also, high dose corticosteroids can lead to premature rupture of membranes. Fetal loss related to the APS usually occurs in the second and third trimesters. Both lupus anticoagulant and aCL are associated with fetal loss in the same. Corticosteroids are currently not advocated for the pregnancy losses due to APS, unless there are allied autoimmune problems.

\section{Neonatal lupus erythematosus (NLE) syndrome}

NLE is a syndrome entailing of Congenital Heart Block (CHB), transient cutaneous lupus lesions, thrombocytopenia, hepatic, and other systemic manifestations in children born to mothers with SLE, Sjogren's syndrome, or other rheumatic diseases with positive anti-Ro/anti-La antibodies. The Research Registry for Neonatal Lupus in the States defines NLE by presence of: (1) maternal antibodies to the $52 \mathrm{kD}$ $\mathrm{SSA} / \mathrm{Ro}, 60 \mathrm{kD} \mathrm{SSA} / \mathrm{Ro}$ or $48 \mathrm{kD} \mathrm{SSB} / \mathrm{La}$ ribonucleoproteins, and (2) $\mathrm{CHB}$ or transient skin rash. ${ }^{15}$ Most manifestations of NLE are transient with the antibodies usually clearing over 6-8 months.

$\mathrm{CHB}$, the most common manifestation of the NLE syndrome $(2 \%)$, is permanent, carrying significant mortality and morbidity to the offspring. ${ }^{16}$ The risk of $\mathrm{CHB}$ upsurges in infants born to women with a previous child having $\mathrm{CHB}$ and occurs in nearly $20 \%$ of pregnancies subsequent to the index pregnancy with CHB. ${ }^{17}$ Occasionally, the mother may be asymptomatic with only $\mathrm{CHB}$ recognition in an infant drawing attention to the presence of anti-Ro/anti-La anti-bodies in mother. The precise pathogenesis of fetal CHB is unknown. The placental passage of maternal autoantibodies during the second trimester, mediating immunological injuries to fetal heart and conduction system, could be responsible. Thus screening for anti Ro/anti La antibodies in first trimester and Fetal echocardiography between 16-24 weeks of gestation can help detecting CHB in utero, besides following the course of the disease and detecting fetal myocarditis, pericardial effusion, and valvular regurgitation. Maternal corticosteroids (which cross the placenta), IVIG and plasmapheresis during second trimester have been attempted to avert placental transfer of harmful pathogenic antibodies, but met with disappointing results. Lupus is a multigenic disorder having a $10 \%$ familial occurrence ${ }^{16}$ with only serologic abnormalities. Thus, Lupus patients run a very low risk of their offsprings developing SLE in later life. 


\section{Immunosuppressive Medications in lupus pregnancy}

While deciding which immunosuppressive medications can be used during pregnancies of women with lupus, risk of maternal disease flare must be prudently weighed against risk of drug exposure to the fetus and induction of miscarriage. In general, it is recommended that immunosuppressive medication required to control lupus disease be continued if possible, particularly in patients with major organ involvement. High dose aspirin and NSAIDs are avoided in the last few weeks of pregnancy because of their effects on uterine contraction, platelet function, and premature closure of ductus arteriosus. ${ }^{18}$ Large doses of salicylates also cause oligohydramnios, and pulmonary hypertension, though fetal anomalies aren't associated..$^{20,21}$

Prednisolone with minimal placental transfer, is the corticosteroid preparation of choice in pregnant lupus patient. With intention to treat the fetus with CHB, steroids crossing the placental barrier like betamethasone, dexamethasone are preferred. High dose corticosteroids carry risk of IUGR, premature membrane rupture, gestational diabetes, hypertension, osteoporosis and avascular bone necrosis. Hydroxychloroquine, the most inoffensive antimalarial used in pregnancy with lupus, ${ }^{19}$ unlike chloroquine, has been reported to cause congenital anomalies. Methotrexate, cyclophosphamide and mycophenolate mofetil is teratogenic and contraindicated in pregnancy. However patients can be safely put on azathioprine and cyclosporine $\mathrm{A}$ when intense immunosuppression is deemed necessary. ${ }^{18}$ Tumor necrosis factor-alpha antagonists are generally discontinued after the first missed period.

\section{Lactation}

Prolactin, having multiple immune-stimulatory effects on the immune system, ${ }^{22}$ normally rises in pregnancy during the second and third trimester, and persists for several months in patients who breast feed. This may contribute to postpartum SLE flares, although the change in the concentrations of other hormones such as oestrogens and progesterone may also be responsible.

Most drugs used for the treatment of SLE are excreted into breast milk. Peak salicylate concentration in milk occurs approximately two hours after the peak serum level. With immature neonatal metabolism, large doses of aspirin, because of fear of salicylate intoxication are best avoided. $^{23}$

NSAIDs with short half-life and inactive metabolites like ibuprofen, indomethacin and naproxen are preferred in nursing mothers, while those with enterohepatic circulation (sulindac) are better avoided. NSAIDs are contraindicated in jaundiced infants because of the fear of kernicterus. Prednisolone and hydroxychloroquine are considered compatible with lactation. Cytotoxics like methotrexate, azathioprine, cyclophosphamide and cyclosporine A are contraindicated in breast feeding mothers due to potential risks of immunosuppression, growth retardation, and carcinogenesis in the neonates. There is still paucity of data on use of dexamethasone or betamethasone in lactating mothers.

\section{TREATMENT RECOMMENDATIONS IN LUPUS}

Mothers with SLE should be assessed for disease activity at least once each trimester and more often if they have active lupus. The schedule for monitoring must include, besides physical examination at the first visit, testing for renal function, complete blood counts, anti-Ro/SSA and anti-La/SSB antibodies, Lupus Anticoagulant (LA) and anticardiolipin antibody (aCL) assays, anti-double stranded DNA antibodies, complement levels (CH50, or $\mathrm{C} 3$ and $\mathrm{C} 4$ ) and uric acid level. ${ }^{24}$

In the absence of any historical feature of APS, patients with SLE and high levels of aCL or the presence of LA must receive low-dose aspirin therapy. Women having both lupus and APS require more frequent monitoring than those with lupus alone. Women who have antibodies to Ro/SSA and/or La/SSB should undergo fetal monitoring for $\mathrm{CHB}$ during 16-24 weeks of pregnancy. There is no therapeutic intervention proven to prevent progression, although early detection may improve outcome. A complete blood count is recommended on a monthly basis during first two trimesters. ${ }^{24}$

At the end of each trimester, glomerular filtration rate, urine protein/urine creatinine ratio, anticardiolipin antibodies (aCL), complement (CH50 or C3 and C4), anti-dsDNA antibodies are recommended. ${ }^{24}$

During the last trimester, in complicated pregnancies, the fetus should be monitored at least weekly using either the biophysical profile score or non-stress test (if no heart block) with amniotic fluid assessment. ${ }^{24}$

Women who show evidence of increased serologic activity but who remain asymptomatic should be monitored more closely. Therapy is not initiated for serologic findings alone. If fetal growth restriction is noted, then umbilical artery Doppler ultrasonography is recommended to monitor fetal well-being. This information helps guide decision making regarding timing of delivery and has been proven to reduce perinatal mortality in this setting.

Women with active disease at conception and those with significant end-organ damage would experience postpartum exacerbations of SLE. ${ }^{25}$ Thus periodic assessment of disease activity is warranted postpartum. With laboratory testing, treatment of postpartum women with active SLE is the same as that of non-pregnant women. However, women who are breast feeding may 
have to stop doing so, depending upon which medications are needed.

\section{CONCLUSION}

Advancing technology and enhanced grasp of the maternal-fetal dyad in lupus have improved outcomes in lupus pregnancies and incidence of SLE exacerbation, especially in those in remission at the beginning of pregnancy. However, hypertensive complications remain a substantial problem. Pregnancy in women with lupus is a high risk pregnancy and judicious monitoring for disease flares and thromboembolic phenomena during the gestation and the puerperal period is mandatory. If planned meticulously, when the disease is quiescent and monitored carefully in a multidisciplinary setting, pregnancy outcomes can be favorable in such patients. In conclusion, pregnancy is no longer considered an absolute contraindication in lupus. Under the vigilant eye of the maternal-fetal medicine specialist and rheumatologist, these women can successfully have children and fulfill their maternal desire.

\section{ACKNOWLEDGEMENTS}

We cannot repay in words, and in deed, love and thanks to all our patients suffering from SLE for their contribution towards making this review reach a successful culmination. We hereby pay our sublime observance and cordial thanks to all of them besides wishing them a speedy recovery.

\section{Funding: No funding sources}

Conflict of interest: None declared

Ethical approval: Not required

\section{REFERENCES}

1. Lockshin MD, Reinitz E, Druzin ML, Murrman M, Estes D. Lupus pregnancy. Case-control prospective study demonstrating absence of lupus exacerbation during or after pregnancy. Am J Med. 1984;77:8938.

2. Urowitz MB, Gladman DD, Farewell VT, Stewart J, McDonald J. Lupus and pregnancy studies. Arthritis Rheum. 1993;36:1392-7.

3. Petri M, Howard D, Repke J. Frequency of lupus flare in pregnancy. The Hopkins Lupus Pregnancy Center experience. Arthritis Rheum. 1991;34:153845.

4. Ruiz-Irastorza G, Lima F, Alves J, Khamashta MA, Simpson J, Hughes GR, et al. Increased rate of lupus flare during pregnancy and the puerperium: a prospective study of 78 pregnancies. Br J Rheumatol. 1996;35:133-8.

5. Mintz G, Niz J, Gutierrez G, Garcia-Alonso A, Karchmer S. Prospective study of pregnancy in systemic lupus erythematosus. Results of a multidisciplinary approach. J Rheumatol 1986;13:732-9.
6. Wong KL, Chan FY, Lee CP. Outcome of pregnancy in patients with systemic lupus erythematosus. A prospective study. Arch Intern Med. 1991;151:26973.

7. McNeil HP, Chesterman CN, Krilis SA. Immunology and clinical importance of antiphospholipid antibodies. Adv Immunol. 1991;49:193.

8. Buyon JP, Kalunian KC, Ramsey-Goldman R, Petri MA, Lockskin MD, Ruiz-Irastorza G, et al. Assessing disease activity in SLE patients during pregnancy. Lupus. 1999;8:677-84.

9. Khamashta MA, Hughes GRV. Pregnancy in systemic lupus erythematosus. Curr Opin Rheumatol. 1996;8:424-9.

10. Petri M. Systemic lupus erythematosus: women's health issues. Bull Rheum Dis. 2000;49:1-3.

11. Mok CC, Wong RWS. Pregnancy in systemic lupus erythematosus. Postgrad Med J. 2001;77:157-65.

12. Buyon JP, Cronstein BN, Morris M, Tanner M, Weissmann G. Serum complement values (C3 and C4) to differentiate between systemic lupus activity and preeclampsia. Am J Med. 1986;21:194-200.

13. Petri M. Pregnancy in SLE. Ballieres Clin Rheumatol. 1998;12:449-76.

14. Martinez-Rueda JO, Arce-Stalinas CA, Kraus A, Alcocer-Varela J, Alarcón-Segovia D. Factors associated with fetal losses in severe systemic lupus erythematosus. Lupus. 1996;5:113-9.

15. Ruyon JP, Hiebert R, Copel J, Craft J, Friedman D, Katholi M, et al. Autoimmune associated congenital heart block, demographics, mortality, morbidity and recurrence rates obtained from a national neonatal lupus registry. J Am Coll Cardiol. 1998;31:1658-66.

16. Meng C, Lockskin M. Pregnancy in lupus. Curr Opin Rheumatol. 1999;11:348-51.

17. Buyon JP, Clancy RM. Neonatal lupus: review of proposed pathogenesis and clinical data from the USbased Research Registry for Neonatal Lupus. Autoimmunity. 2003;36:41-50.

18. Lockshin MD, Sammaritano LR, Schwartzman S. Lupus pregnancy. In: Lahita RG, eds. Systemic Lupus Erythematosus. 3rd ed. New York: Academic Press; 1999: 507-536.

19. Ramsey-Goldman R, Schilling E. Immunosuppressive drug use during pregnancy. Rheum Dis Clin North Am. 1997;23:149-67.

20. Turner G, Collins E. Fetal effects of regular salicylate ingestion in pregnancy. Lancet. 1975;ii:338-9.

21. Jick H, Holmes LB, Hunter JR, Madsen S, Stergachis A. First-trimester drug use and congenital disorders. JAMA. 1981;246:343-6.

22. Reber PM. Prolactin and immunomodulation. Am J Med. 1993;295:637-44.

23. Committee on Drugs, American Academy of Pediatrics. The transfer of drugs and other chemicals into human milk. Pediatrics. 1994;93:137-50.

24. Lockshin MD, Sammaritano LR. Lupus pregnancy. Autoimmunity. 2003;36:33. 
25. Andrade RM, McGwin G Jr, Alarcón GS, Sanchez ML, Bertoli AM, Fernández M, et al. Predictors of post-partum damage accrual in systemic lupus erythematosus: data from LUMINA, a multiethnic US cohort (XXXVIII). Rheumatology (Oxford). 2006;45:1380-4.
DOI: 10.5455/2320-1770.ijrcog20150402

Cite this article as: Mohindra R, Marwah S. Systemic lupus erythematosus in pregnancy - intricate, but wieldy. Int J Reprod Contracept Obstet Gynecol 2015;4:295- 